\title{
Phylogenetic Consensus for Exact Median Trees
}

\author{
Extended Abstract
}

\author{
Paweł Tabaszewski \\ Faculty of Mathematics, Informatics \\ and Mechanics, University of Warsaw \\ Warsaw, Poland \\ pt335957@students.mimuw.edu.pl
}

\author{
Paweł Górecki \\ Faculty of Mathematics, Informatics \\ and Mechanics, University of Warsaw \\ Warsaw, Poland \\ gorecki@mimuw.edu.pl
}

\author{
Oliver Eulenstein \\ Department of Computer Science, \\ Iowa State University \\ Ames, IA, USA \\ oeulenst@iastate.edu
}

\begin{abstract}
Solving median tree problems is a classic approach for inferring species trees from a collection of discordant gene trees. Such problems are typically NP-hard and dealt with by local search heuristics. Unfortunately, such heuristics generally lack any provable correctness and precision. Algorithmic advances addressing this uncertainty, have led to exact dynamic programming formulations suitable to solve a well-studied group of median tree problems for smaller phylogenetic analyzes. However, these formulations allow to compute only very few optimal species trees out of possibly many such trees, and phylogenetic studies often require the analysis of all optimal solutions through their consensus tree. Here, we describe a significant algorithmic modification of the dynamic programming formulations that compute the cluster counts of all optimal species trees from which various types of consensus trees can be efficiently computed. Through experimental studies, we demonstrate that our parallel implementation of the modified programming formulation is more efficient than a previous implementation of the original formulation, and can greatly benefit phylogenetic analyses.
\end{abstract}

\section{KEYWORDS}

consensus tree, median tree, exact solution, gene tree parsimony, duplication cost

\section{ACM Reference Format:}

Paweł Tabaszewski, Paweł Górecki, and Oliver Eulenstein. 2018. Phylogenetic Consensus for Exact Median Trees: Extended Abstract. In ACM-BCB'18: 9th ACM International Conference on Bioinformatics, Computational Biology and Health Informatics, August 29-September 1, 2018, Washington, DC, USA. ACM, New York, NY, USA, 10 pages. https://doi.org/10.1145/3233547.3233560

\section{INTRODUCTION}

Phylogenetic tree estimation is progressively enriching our understanding of the way in which the historical relationships of evolutionary entities (e.g., molecular sequences, genomes, and species) have evolved. An understanding of the fundamental principles that explain how such entities have evolved is largely benefitting the biological oriented research areas $[12,17,27]$. The knowledge of

Permission to make digital or hard copies of all or part of this work for personal or classroom use is granted without fee provided that copies are not made or distributed for profit or commercial advantage and that copies bear this notice and the full citation on the first page. Copyrights for components of this work owned by others than ACM must be honored. Abstracting with credit is permitted. To copy otherwise, or republish, to post on servers or to redistribute to lists, requires prior specific permission and/or a fee. Request permissions from permissions@acm.org.

ACM-BCB'18, August 29-September 1, 2018, Washington, DC, USA

(C) 2018 Association for Computing Machinery.

ACM ISBN 978-1-4503-5794-4/18/08 . .\$15.00

https://doi.org/10.1145/3233547.3233560 these principles is increasingly used to make predictions about how evolutionary entities may evolve in the future. Thus, phylogenetic trees are not only fundamental to biology, but can also be powerful predictors that are aiding numerous other research disciplines, including epidemiology, human health, conservation biology, and linguistics [17-19, 27].

Despite these promises, phylogenetic tree estimation is one of the most challenging problems in evolutionary biology. Traditionally, a phylogenetic tree is estimated by first sampling a single gene (i.e., a gene family) for a set of given species, and then by inferring the evolutionary history of this gene, which is called a gene tree. Genes are part of the species genomes, and therefore, it is assumed that the gene tree is mimicking the evolution of the given species. Thus, the gene tree is identified with the species tree. Unfortunately, distinct gene trees sampled from the same set of species can have discordant evolutionary relationships due to complex evolutionary processes affecting genomic locations differently [8, 29]. Even in the absence of such evolutionary events, there can be discord in the gene trees that is attributable to error in the tree inference process and noise in the underlying data. Error and evolutionary processes causing discordance can occur on a staggering scale [20,28]. Hence, identifying gene trees with their corresponding species trees can result in largely inaccurate species trees.

Solving median tree problems has become a powerful approach to infer a species tree from a given collection of possibly discordant gene trees [2]. A median tree problem seeks, given set of gene trees, a species tree (i.e., a median tree) that is minimizing the overall cost to the in input trees using a problem-specific cost function. For instance, the classic tree reconciliation cost functions account for the minimum number of evolutionary events (e.g., gene duplications, gene losses, gene duplications plus losses [3, 11, 13, 15], and deep coalescence [22]) that can explain the discord between a gene tree and a species tree. Another example would be metrics that measure the error between a gene tree and a species tree, such as the popular Robinson-Foulds (RF) cost [4, 32].

Median tree problems used in practice are NP-hard [2] and therefore are addressed by local search heuristics [1,34], which have produced a few credible species tree estimates [7, 23, 30]. However, such heuristics generally lack provable accuracy and precision $[6,26]$, since their task is to efficiently find a globally optimal species tree in a highly complex solution landscape whose size is double factorial in the size of the searched tree. Moreover, this landscape has typically numerous local optima that can trap heuristic approaches.

To address such heuristic uncertainties, exact dynamic programming algorithms for a well-studied group of classic NP-hard median 
tree problems that are based on tree reconciliation costs and the RF cost have been developed by Chang et al. [6]. These algorithms are significantly faster than the naïve enumeration of the solution space and allow computing optimal species trees for smaller phylogenetic studies. Unfortunately, there is a major drawback preventing their general applicability in practice - that is - they can compute only very few optimal species trees out of possibly many such trees for a given problem instance. Practitioners, on the other hand, are generally interested in analyzing all of the optimal species trees through their representation as a consensus tree [5].

Here we provide essential and non-trivial modifications of the existing dynamic programming formulations from Chang et al. to compute without enumerating all optimal solutions the frequency count of the clusters (clades) over all exact solutions. From this count, various types of consensus trees are linear time computable. We provide an efficient parallel implementation of our modified formulations that is much more efficient and effective than the implementation of the original formulation from Chang et al., which we demonstrate through a scalability study and an empirical analysis.

\subsection{Related work}

In this section, we overview the median tree problems that are based on the classic reconciliation costs and the RF cost.

Tree reconciliation costs are an essential aspect of a process called tree reconciliation that has been introduced by the pioneering work of Goodman et al. nearly 40 years ago [13]. Given a gene tree and a species tree, this process is embedding the gene tree into the species tree through a mapping function. This mapping relates every gene in the gene tree to the most recent species in the species tree, referred to as its host-species that could have contained the gene. For example, the mapping relates every leaf-gene of the gene tree to the leaf-species in the species tree from which it has been sampled. Based on this mapping the evolutionary events of gene duplication, gene loss, and deep coalescence are identified [10,29]. Gene duplication is a gene in the gene tree that has the same host-species as at least one of its children, and a gene loss is accounted for by each maximum subtree in the species tree that has no mapping from the gene tree. The reconciliation costs associated with these events are termed gene duplication cost, gene loss cost, and gene duplication plus loss cost accounting for the number of gene duplications, gene losses, and gene duplications plus losses, respectively. The deep coalescence cost is another classic reconciliation cost that has been introduced by Maddison [22]. This cost accounts for every edge in the species tree the number of embedded gene tree lineages minus one, which are assumed to be caused by the deep coalescence events.

The Robinson-Foulds (RF) cost was introduced by Bourque [4] and later on refined by Robinson and Foulds [32]. This cost is defined for two, in our case binary, rooted trees over the same label set, as the cardinality of the symmetric difference of the cluster representation of the trees. A cluster for a vertex in a rooted tree is defined to be the set of all leaf-labels of the subtree rooted at this vertex, and the cluster representation of a tree is the set of the clusters for each vertex in the tree.
The median tree problems under the presented reconciliation costs and the RF cost can be computed exactly in $O\left(3^{n} \mathrm{mn} / \mathrm{b}\right)$ time and $O\left(2^{n}+m\right)$ space, using dynamic programming, a bit-vector of size $b$ (e.g., representing the register size of the computational machine used) and Gray encoding, where $n$ is the overall number of taxa presented in input trees, and $m$ is the number of unique rooted splits in the input gene trees [6].

Given a set of rooted and binary input trees over the same label set, the strict consensus tree [25] and majority consensus tree [24] for these input trees is the tree that consists exactly of the clusters that occur in every input tree and more than half of the input trees respectively. All of the trees involved have the same cluster for their roots and the same set of clusters for their leaves. These clusters are referred to as trivial clusters. The clusters of the majority consensus tree are referred to as majority clusters.

\subsection{Contribution}

We describe a non-trivial modification of the dynamic programming formulations from Chang et al. [6] that, for the first time, computes the overall cluster counts of all optimal species trees (rather than few optimal species trees), by avoiding their individual computation. Every type of consensus tree that is defined based on cluster counts can be efficiently computed from these counts, including the strict-, majority-, and greedy-consensus tree. While our modification of the original dynamic programming formulations causes an increase in algorithmic runtime, it does not increase the original asymptotic upper bound on the runtime.

We implemented a parallelized version of our improved dynamic programming formulations utilizing standard techniques for efficiency, such as bit-vector and Gray encodings. Through a scalability study, we demonstrate that our implementation is much more time efficient than the implementation of the original dynamic programming formulation from Chang et al. [6]. In fact, our implementation can handle nearly any number (e.g., millions) of optimal solutions for smaller sized studies. Finally, we illustrate through an empirical study the correctness and applicability of our parallel implementation. In addition, this study allowed us to correct a consequential analytical result from the empirical study presented by Chang et al. that was caused by an overflow error in the original implementation.

\section{PRELIMINARIES}

We review basic definitions, concepts, and terminology from computational phylogenetics that are needed in the context of this work.

\subsection{Basic definitions and terminology}

A gene tree $G$ is a rooted full binary tree having leaves labeled by species names. By $L(G)$ we denote the set of all labels present in $G$. A node $n$ is called internal if it has two children. A cluster for a node $g$ in a rooted tree, denoted by $\operatorname{clu}(g)$, is defined to be the set of all leaf-labels of the subtree rooted at this node. A species tree is a gene tree with distinctly labeled leaves. By path $(x, y)$ we denote the set of all nodes on the shortest path connecting nodes $x$ and $y$.

Let $S$ be a species tree. By $a+b$ we denote the least common ancestor of nodes $a$ and $b$ in $S$. We use the partial order relation 
$a \leq b$ if $b$ is a node on the path between $a$ and the root of $S$. For a gene tree $G$ where $L(G) \subseteq L(S)$, a least common ancestor (lca) mapping, is a function $M$ from the nodes of $G$ to the nodes of $S$, where $M(g)=s$ if $g$ and $s$ are leaves with the same label, or $M(g)=M(a)+M(b)$ if $a$ and $b$ are the children of $g$.

\subsection{Cost functions}

We define five classical functions, called here costs, used to compare a gene tree and a species tree: duplication $(D)$, deep coalescence $(D C)$ [22], loss $(D)$, duplication-loss $(D L)$ and Robinson-Foulds $(R F)$ [32]. Each of these costs is linear time and space computable [9, 21]. For a cost $c \in\{D, D C, L, D L, R F\}$, let $\xi^{c}: V_{G} \times V_{S} \rightarrow R$ be a contribution function defined as follows:

- $\xi^{D}(g, s)=\mathbb{1}\left[\exists g^{\prime} \in \mathrm{Ch}(g)\right.$ and $\left.M(g)=s=M\left(g^{\prime}\right)\right]$,

- $\xi^{L}(g, s)=\mathbb{1}\left[\left\{g^{\prime}, g^{\prime \prime}\right\}=\operatorname{Ch}(g)\right.$ and $\left.s \in \operatorname{In}\left(M\left(g^{\prime}\right), M\left(g^{\prime \prime}\right)\right)\right]$,

- $\xi^{D C}(g, s)=\mathbb{1}\left[\left\{g^{\prime}, g^{\prime \prime}\right\}=\mathrm{Ch}(g)\right.$ and $M(g) \neq s \in \operatorname{path}\left(M\left(g^{\prime}\right)\right.$, $\left.\left.M\left(g^{\prime \prime}\right)\right)\right]$

- $\xi^{R F}(g, s)=2 * \mathbb{1}[M(g)=s$ and $\operatorname{clu}(g) \neq \operatorname{clu}(s)]$,

- and $\xi^{D L}(g, s)=\xi^{D}(g, s)+\xi^{L}(g, s)$,

where $\operatorname{Ch}(g)$ is the set of children of $g, \mathbb{1}$ is the indicator function and $\operatorname{In}(v, w)$ is the set of loss nodes between $v$ and $w$ defined as follows. If $v \leq w$, then $\operatorname{In}(v, w)=\operatorname{path}(v, w) \backslash\{v\}$. If $w \leq v$, then $\operatorname{In}(v, w)=\operatorname{path}(v, w) \backslash\{w\}$. Finally, $\operatorname{In}(v, w)=\operatorname{path}(v, w) \backslash$ $\{v, w, v+w\}$ if $v$ and $w$ are incomparable. Then, the cost of reconciling a gene tree $G$ and a species $S$ under $c$ equals $c(G, S)=$ $\sum_{g \in G, s \in S} \xi^{c}(g, s)$ (the reader is referred to $[6,14]$ for a detailed treatment).

\subsection{Problems}

We write that a tree $S$ is over a set of species $I$ if $L(S)=I$. Let $Q$ be a collection of gene trees. By $L(Q)$ we denote the set of all species present in trees of $Q$. Now, we extend the notion of the cost function to collections of gene trees. For a given $S$ over $L(Q)$, by $c(Q, S)$ we denote the total cost $\sum_{G \in Q} c(G, S)$.

Finally, we can define the following problem.

Problem 2.1. [MinST - Minimal Species Tree] Given a collection of gene trees $Q$ and a cost function c find a species tree $S^{\min }$ that minimizes $c(Q, S)$ in the set of all species trees $S$ over $L(Q)$.

The species tree $S^{\text {min }}$ will be called minimal (for $Q$ and $c$ ) and the minimal cost we denote by $c^{\min }(Q)$.

Problem 2.2. [MinCC - Minimal Cost Computation] Given a collection of gene trees $Q$ and a cost function c compute $c^{\min }(Q)$.

The MinCC problem is NP-hard under $D$ [21] and $D C$ [35].

\subsection{Dynamic Programming Solution}

A dynamic programming solution for the MinCC problem was proposed in [6]. Here, we recall the basic formulas needed to compute minimal trees [6]. If $g$ is an internal node of a tree whose children have clusters $A$ and $B$, respectively, then by $A \mid B$ we denote the rooted split of $g$. If $T$ is a tree, then by $r(T)$ we denote the multiset of all rooted splits present in $T$. Similarly, we denote $r(Q)$ as $\bigcup_{T \in Q} r(T)$ if $Q$ is a set of gene trees.

For a species leaf $s$ and a split $X \mid Y$, let the total cost contribution functions for our standard costs be defined as follows. For $c \in$
$\{D, L, D C, R F\}$ (DL is a sum of $\mathrm{D}$ and $\mathrm{L}$, and thus omitted)

$$
\Lambda^{c}(Q, s)=\sum_{q \in r(Q)} \lambda^{c}(q, s)
$$

and

$$
\Gamma^{c}(Q, X \mid Y)=\sum_{q \in r(Q)} \gamma^{c}(q, X \mid Y)
$$

where

- $\lambda^{D}(A \mid B, s)=\mathbb{1}[A \cup B=\{s\}]$,

- $\lambda^{L}(A \mid B, s)=\lambda^{R F}(A \mid B, s)=0$,

- $\lambda^{D C}(A \mid B, s)=\mathbb{1}\left[\exists i: A_{i}=\{s\} \neq A_{i+1}\right]$,

- $\gamma^{D}\left(A_{1}\left|A_{2}, X_{1}\right| X_{2}\right)=\mathbb{1}\left[A_{1} \cup A_{2} \subseteq X_{1} \cup X_{2} \wedge \exists i: X_{1} \nsupseteq A_{i} \nsubseteq\right.$ $\left.X_{2}\right]$,

- $\gamma^{L}\left(A_{1}\left|A_{2}, X_{1}\right| X_{2}\right)=\mathbb{1}\left[\exists i, j: A_{i} \subseteq X_{j} \wedge X_{1} \nsupseteq A_{i+1} \nsubseteq X_{2}\right]$,

- $\gamma^{D C}\left(A_{1}\left|A_{2}, X_{1}\right| X_{2}\right)=\mathbb{1}\left[\exists i, j: X_{j} \nsupseteq A_{i} \subseteq X_{1} \cup X_{2} \nsupseteq\right.$ $\left.A_{1+(i \bmod 2)}\right]$,

- and $\gamma^{R F}\left(A_{1}\left|A_{2}, X_{1}\right| X_{2}\right)=2 \cdot \mathbb{1}\left[A_{1} \cup A_{2} \subsetneq X_{1} \cup X_{2} \wedge \forall i:\left(A_{1} \cup\right.\right.$ $\left.\left.A_{2}\right) \cap X_{i} \neq \emptyset\right]$.

In other, words $\Lambda^{c}(Q, s)$ denotes the contribution of a leaf $s$ to the cost $c(Q, S)$, when reconciling $Q$ with any species tree $S$ over $L(Q)$. Similarly, $\Gamma(Q, X \mid Y)$ is the contribution of an internal node $x$ having split $X \mid Y$ of some species tree $S$ over $L(Q)$ when reconciling $Q$ with $S$.

We have the following property that establishes a relation between a contribution function and the total cost contribution function.

Lemma 1 (From [6]). Let $Q$ be a set of gene trees, $c$ be a cost function and $S$ be a species tree over $L(Q)$. Then,

$$
c(Q, S)=\sum_{s \in L(S)} \Lambda^{c}(Q, s)+\sum_{X \mid Y \in r(S)} \Gamma^{c}(Q, X \mid Y) .
$$

Let $Z \subseteq L(Q)$. Then, dynamic programming formula $\Delta^{c}$ for MinCC is defined as follows (the superscript $c$ is omitted in $\Delta, \Gamma$ and $\Lambda)[6]$ :

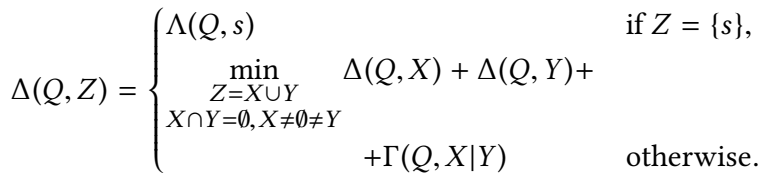

Then, for any cost function $c$, we have $\Delta^{c}(Q, L(Q))=c^{\min }(Q)$ (see Theorem 1 from [6]). Finally, solving MinST follows from (1) by backtracking applied to the values of $\Delta$. The algorithm requires $O\left(3^{n} m n / b\right)$ time and $O\left(2^{n}+m\right)$ space using a bit-vector of size $b$ and Gray encoding, where $n$ and $m$ are the number of species and unique rooted splits represented in the input trees, respectively [6].

\section{RESULTS}

To infer consensus trees from all optimal solutions we need to compute for each cluster the number of minimal trees in which the cluster is present. First, we show the formula for cluster counts, then, based on the formula, we describe an algorithm for the computation of counts. 


\subsection{Counting Clusters in Exact Solutions}

The set of all splits $X \mid Y$ of a non-singleton set $Z$ for which $\Delta(Q, Z)=$ $\Delta(Q, X)+\Delta(Q, Y)+\Gamma(Q, X, Y)$ in (1) is denoted OptSpl $(Z)$. By $\mathrm{\top}$ we denote the top cluster of a species tree, i.e., the set of all species present in the input collection $Q$. Let

$$
\Omega(Z)= \begin{cases}1 & \text { if } Z=\{s\}, \\ \sum_{X \mid Y \in \mathrm{OptSpl}(Z)} \Omega(X) \Omega(Y) & \text { otherwise. }\end{cases}
$$

A subtree in a tree $T$ whose root is cluster $Z$ will be called $Z$ subtree (of T).

Lemma 2. For any $Z \subseteq \mathrm{T}, \Omega(Z)$ is the total number of nonisomorphic $Z$-subtrees present in minimal trees.

Proof. It follows by induction on the size of $Z$.

CONCLUSion 1. The number of minimal trees equals $\Omega(T)$.

Observe that the occurrence count of a cluster $X$ in all solutions is generally not computable using $\Omega$. The value $\Omega(Q, X)$ depends only on splits of subsets of $X$. Thus, if $X$ is a not the top cluster, then $\Omega(Q, X)$ does not include the variants in the rest of the tree outside $X$. For example, see the cluster $b d$ in Figure 1 .

Let $\#(X)$ denote the occurrence count of a cluster $X$ in all solutions.

Finally, we can present the main theoretical result.

Theorem 3.1 (Cluster Counts in Optimal Solutions). For every non-empty $X \subseteq \top$

$\#(X)= \begin{cases}\Omega(T) & \text { if } X=\top \\ \sum_{Y \text { such that } X \mid Y \in \operatorname{OptSpl}(X \cup Y)} \frac{\Omega(X) \Omega(Y) \#(X \cup Y)}{\Omega(X \cup Y)} & \text { otherwise. }\end{cases}$

Proof. Let $T$ be a minimal tree. Given a node $v \in T$, whose cluster is $Z$, we can infer from $T, \Omega(Z)$ non-isomorphic minimal trees by replacing the subtree rooted at $v$ with each $Z$-subtree. Thus, for every $Z$, \# $(Z) / \Omega(Z)=\alpha_{Z}$ is an integer, which also denotes how many times $\Delta(Q, Z)$ is visited when reconstructing all optimal solutions from (1).

Let $X \mid Y \in \operatorname{OptSpl}(X \cup Y)$. Then $\Omega(X) \Omega(Y)$ is the number of non-isomorphic $X \cup Y$-subtrees such that the children of root have clusters $X$ and $Y$, respectively. Now, if we know $\alpha_{X \cup Y}$, then the total number of occurrences of $X$ in minimal trees such that $Y$ is a sibling cluster of $X$ equals $\Omega(X) \Omega(Y) \alpha_{X \cup Y}$.

\subsection{Algorithm}

Algorithm 1 shows how to compute values of \# $(X)$ for every cluster using OptSpl. When a cluster $X$ is processed, it is required that $\#(Z)$ is computed, for every $Z$ such that $X \subset Z$ (see the presence of \# $(X \cup Y)$ in \# formula (3) in Theorem 3.1). This requirement is reflected in the 7-th line of Algorithm 1 by processing clusters in size-descending order. The time complexity is linear in the size of the OptSpl structure that is exponential in the number of species.

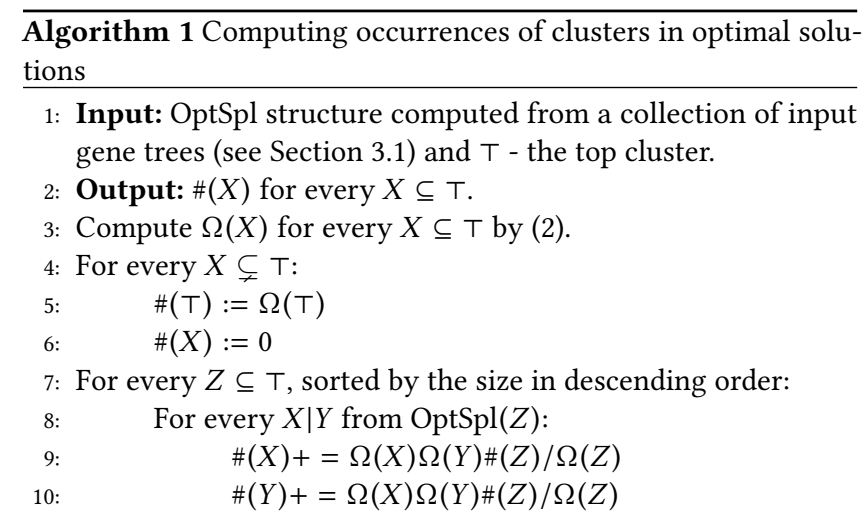

\subsection{Consensus Trees from Cluster Counts}

Now, computing the most popular types of consensus trees, that is, majority-, strict- or greedy-consensus, is straighforward given the cluster counts provided by our algorithm. For example, for majority consensus build a tree from all clusters $X$, such that $\#(X)>\frac{1}{2} \Omega(T)$, while for strict consensus take clusters such that $\#(X)=\Omega(T)$. See also [5].

\subsection{Maximal (Worst) Trees}

Analogously to Problems 2.1 and 2.2 we define the worst-case variants by replacing "min" with "max" in the definitions. Species trees maximizing the total cost are called maximal or worst. The maxproblems can be solved through our dynamic programming approach by replacing min with max in Formula 1 [6]. Observe that the consensus trees from all maximal trees can be inferred by using the approach presented in the previous paragraph.

\subsection{Parallel Implementation}

Our algorithm was parallelized with OpenMP, where clusters of the same size can be processed in parallel, which achieves a near perfect strong scaling. Further efficiency was achieved by using standard bit-vector and Gray encodings, and by vectorizing the cost functions via intrinsics. Next, splits with $\#(X)=0$ were omitted, which provides a significant speed-up when the number of optimal trees is small. The program is written in C using gcc-5.4.0.

\section{EXPERIMENTAL EVALUATION}

We demonstrate the ability of our parallel implementation through a scalability analysis and an empirical study. For convenience, by classic costs we are referring to the costs based on gene duplications (D), losses (L), duplications plus losses (DL), deep coalescences (DC), and Robinson-Foulds (RF). The studies were performed using an i7-6850K processor with 6 cores/ 12 threads and 64GB DDR4. Note that the size of the RAM is not crucial here, since all splits from our datasets could be stored in L2 CPU cache.

\subsection{Scalability Analysis}

We analyze the runtime performance of our parallel implementation for the modified dynamic programming formulation in practice. Our hypothesis is that this runtime corresponds smoothly to the asymptotic runtime of the algorithm and that our implementation 

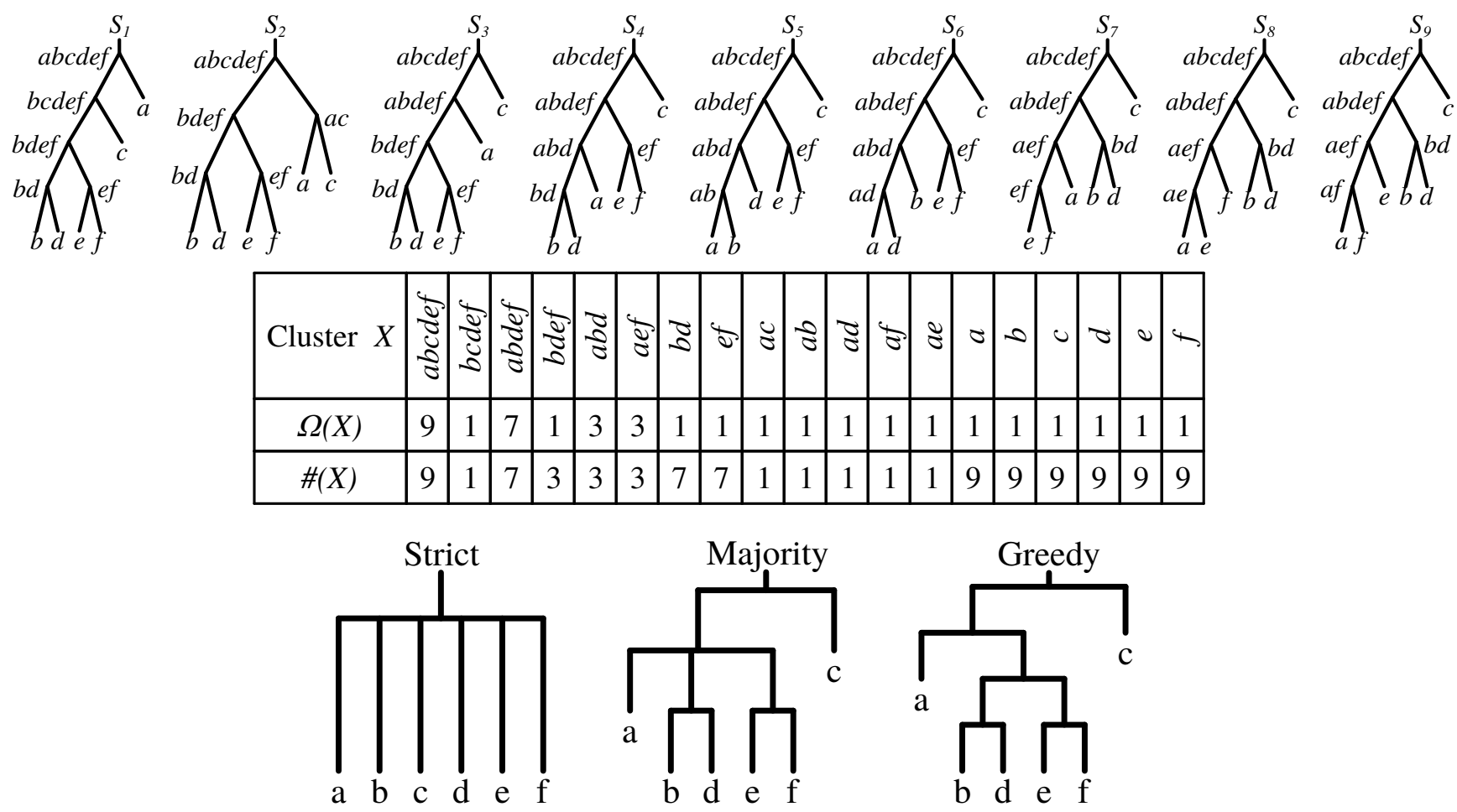

Figure 1: An example of exact solutions. Top: All nine minimal species trees $S_{1}-S_{9}$ having the lowest duplication cost of 1 when solving MinST with the input consisting of $Q=\{((a, b),(((b, c), d), b))\}$. Middle: All 19 clusters present in $S_{1}-S_{9}$ with values of $\Omega$ and \#. Bottom: Consensus trees obtained from exact solutions using cluster frequencies from \#. Note that the greedy consensus tree is non-unique.

is more efficient than the implementation from Chang et al. [6] of the original programming formulation.

4.1.1 Data. We created our dataset from the TreeFam v9.0 [33] database consisting of 1279 gene family trees from overall 109 species. From these species, we selected randomly five sets, each of size $N$, for every $N \in\{10,11, \ldots, 18\}$. For each of these resulting 27 species sets, say $X$, we obtained the set of trees consisting of the by $X$ induced subtree of every tree in TreeFam.

4.1.2 Experimental Setting. Our implementation was run for every tree in the created dataset for each of the classic costs. Then the average runtime was reported for the trees with $N$ leaves, for every $N \in\{10,11, \ldots, 18\}$, which are depicted in Figure 2 (top). Since the runtime of the algorithm also depends on the number of splits (in addition to the overall number of taxa), we reported their average number for the trees of size $N$ in the input dataset in Figure 2 (bottom).

4.1.3 Results and Discussion. From Figure 2 we observe that the average runtime grows exponentially in the overall number of taxa in the input trees for each of the classic costs. Thus the observed practical runtimes follow the asymptotic upper bound of the implemented algorithms. Further, the growth rates under the classic cost functions are increasing in the order D, DC, L, RF, and DL reflecting the increasingly larger constant factors involved in computing solutions under these costs, e.g., the runtime for DL is the additive runtime for $\mathrm{D}$ and $\mathrm{L}$.

Now we discuss how the runtimes of our implementation compare to the runtimes of the previously, up to our knowledge, fastest implementation for computing exact solutions under the classic costs [6]. From Table 1 in [6] that depicts the runtimes of the previous implementation, we observe that the runtimes of both implementations in terms of the number $N$ of overall taxa of the input trees is similar. However, the datasets for our implementation handle 1000 trees for every $N$, while the previous implementation was run on datasets with only 100 trees. More precisely, the runtimes depend on the overall number of distinct rooted splits in the input trees. For our input datasets their corresponding number of splits is summarized in Figure 2 (bottom). In addition, our implementation provides also the cluster counts of all optimal solutions, requiring extra algorithmic steps. Therefore, in comparison to the previous implementation, our implementation computes much larger datasets and provides more information for the same runtime when measured in the overall taxon number $N$. In conclusion, our implementation is significantly faster than the previous implementation, which is due to the parallel implementation of our algorithms described in Section 3.5. 

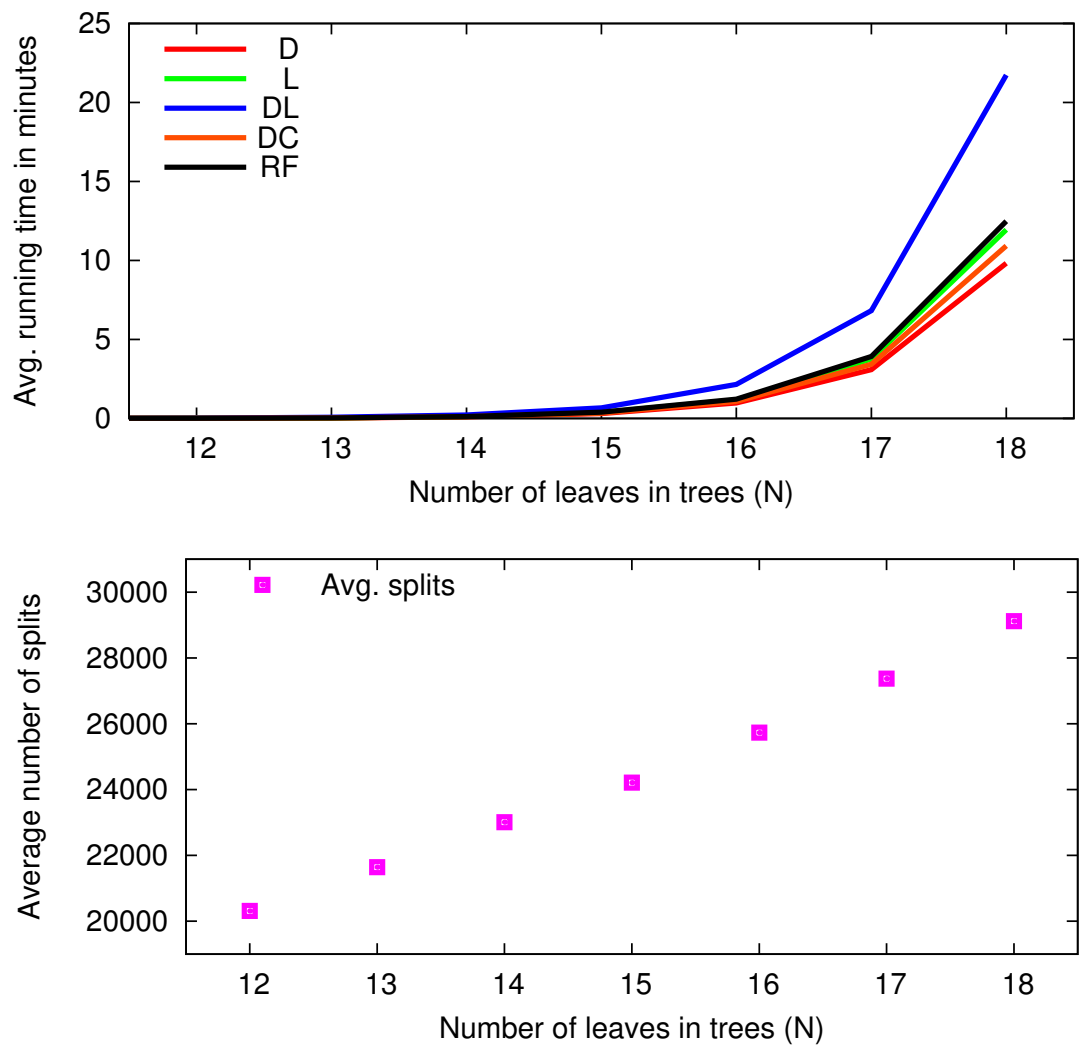

Figure 2: Top: Running time for datasets sampled from TreeFam. Bottom: The average number of splits present in the input datasets.

\subsection{Empirical Study}

We demonstrate the ability and correctness of our implementation using the Guigó et al. dataset [16] for which all solutions are known [6,31].

4.2.1 Data. We re-evaluated the dataset from Guigó et al. consisting of 53 distinct gene trees from overall 16 major higher eukaryotic taxa.

4.2.2 Experimental Setting. Running our implementation on the dataset reported several analytic values for the set of all solutions (i.e., the minimum scoring species trees) under each of the classic costs. For each set of solutions the following values were reported:

(i) the minimal cost of a tree in the set,

(ii) the size of the set,

(iii) the strict-, majority-, and greedy-consensus of the trees in the set,

(iv) and the Majority cluster counts for the minimal treesof the trees in the set.

The results of (i)-(iii) are depicted in Figure 3, and for (iv) are summarized in Table 1. Similarly, we computed the analytic values for each set of all maximum scoring species trees, which are summarized in Figure 3 and Table 2. The total runtime to obtain all of the reported values was approximately six seconds.
4.2.3 Results and Discussion. The optimal (minimum) scores and the number of optimal solutions in Figure 3 confirm the corresponding results presented in Table 2 [6]. However, our efficient implementation computed these results nearly 17 times faster than the previous implementation [6]. In addition, our implementation computed the cluster counts for each set of optimal solutions and inferred from these counts three popular types of consensus trees. The 12 optimal trees for the Guigo et al. dataset under the DL cost have been originally computed by Page and Charleston [31], confirming our cluster count for these trees. However, consensus counts for the solutions under the remaining classic costs were not previously available. Here, for the first time, we are analyzing these counts and their corresponding consensus trees. From Table 1 we observe that most of the majority consensus clusters occur in all of the solution trees for the biologically motivated costs D, L, DL, and DC. This suggests that the solutions under the biological costs have a "stronger" consensus than under the RF cost. Furthermore, we confirmed that the solutions under $\mathrm{L}$ and DL are the same, which suggest further investigation into the relationship between these two costs. Consequently, these costs produce the same majority consensus clusters and the same strict-, majority-, and greedy consensus trees (see Figure 3). Comparing the consensus trees in Figure 3 we observe that the strict consensus trees under D (for 71 trees) and L/DL (for 12 trees) are the same, but then the refinements 
of these trees for the majority- and greedy-consensus differ. While the consensus trees under DC share some "similarities" with the consensus trees under $\mathrm{D}$ and $\mathrm{L} / \mathrm{DL}$, the consensus trees under $\mathrm{RF}$ are quite different and also less resolved, which might be caused by the large number of solutions (i.e., 1291 trees). These observations support the hypothesis that the discord in the gene trees of the Guigo et al. data set is largely due to gene duplications and losses as originally suggested by Guigo et al. [16].

Identifying worst case clusters could be beneficial when solving problems with the exploration of the species tree space, for instance, in supertree inference. Also, this allows evaluating the min result, e.g., clusters that are frequent in both the minimal and the maximal solutions might be irrelevant for the biological analysis. The worst scores and the number of optimal solutions in Figure 4 confirm the results previously presented in [6] for all classic costs, but not the RF cost (i.e., 15, 971, 496, 451, 840 trees versus 2, 808, 045, 312 trees in [6]). We found that this discrepancy is due to an overflow error in the previous implementation, which largely underestimated the count of the worst case trees under RF.

\section{CONCLUSION}

There is an increased interest in smaller phylogenetic studies using exact solutions for NP-hard median tree problems. When faced with several such solutions practitioners are interested in analyzing their consensus tree, and there are various well-studied types of such trees available. Here, we presented the first exact algorithm that allows computing such consensus trees for smaller studies under several classic NP-hard median tree problems. While enumerating all solutions becomes prohibitive for such studies, our algorithm is directly computing the cluster counts over all solutions. In fact, this direct approach can handle nearly any number of optimal solutions for smaller sized studies. To further increase the size of phylogenetic studies of consensus trees, we used a highly efficient parallel implementation of our algorithm. Our studies have demonstrated the ability of this implementation and provided the first consensus trees for the well-researched Guigo et al. dataset that can have several hundred solution trees depending on the chosen median tree problem. The presented parallel implementation provides a new tool for practitioners to infer and compare various types of consensus trees under classic median tree problems.

\section{Acknowledgments}

This material is based upon work supported by the National Science Foundation under Grant No. 1617626 and by National Science Centre grant No. 2015/19/B/ST6/00726.

\section{REFERENCES}

[1] Mukul S. Bansal and Oliver Eulenstein. 2013. Algorithms for genome-scale phylogenetics using gene tree parsimony. IEEE/ACM Trans. Comput. Biol. Bioinform. 10, 4 (2013), 939-56.

[2] Olaf R.P. Bininda-Emonds, John L. Gittleman, and Mike A. Steel. 2002. The (Super)Tree of Life: Procedures, Problems, and Prospects. Annu. Rev. Ecol. Evol. Syst. 33, 1 (2002), 265-289.

[3] Paola Bonizzoni, Gianluca Della Vedova, and Riccardo Dondi. 2005. Reconciling a gene tree to a species tree under the duplication cost model. Theor. Comput. Sci. 347, 1-2 (2005), 36-53.

[4] Michel Bourque. 1978. Arbres de Steiner et reseaux dont certains sommets sont a localisation variable. Ph.D. Dissertation. University de Montreal, Montreal, Quebec.
[5] David Bryant. 2003. A classification of consensus methods for phylogenetics. DIMACS Series in Discrete Mathematics and Theoretical Computer Science 61 (2003), 163-184.

[6] Wen-Chieh Chang, Paweł Górecki, and Oliver Eulenstein. 2013. Exact solutions for species tree inference from discordant gene trees. F. Bioinform. Comput. Biol. 11, 5 (2013), 1342005.

[7] James A. Cotton and Roderic D.M. Page. 2002. Going nuclear: gene family evolution and vertebrate phylogeny reconciled. Proc. Biol. Sci. 269, 1500 (2002), 1555-61.

[8] James A. Cotton and Roderic D.M. Page. 2005. Rates and Patterns of Gene Duplication and Loss in the Human Genome. Proc. R. Soc. Lond. B. Biol. Sci. 272, 1560 (2005), 277-83.

[9] William H.E. Day. 1985. Optimal algorithms for comparing trees with labeled leaves. Cladistics - The International fournal of the Willi Hennig Society 2, 1 (1985), 7-28.

[10] Oliver Eulenstein. 1998. Vorhersage von Genduplikationen und deren Entwicklung in der Evolution. Ph.D. Dissertation. Bonn University, 53012 Bonn, Germany.

[11] Oliver Eulenstein, Snehalata Huzurbazar, and David A. Liberles. 2010. Evolution after Gene Duplication. John Wiley, Chapter Reconciling Phylogenetic Trees.

[12] Peter Forster and Colin Renfrew. 2006. Phylogenetic methods and the prehistory of languages. McDonald Inst. of Archeol. Reas., Cambridge, England, United Kingdom.

[13] Morris Goodman, John Czelusniak, G. William Moore, A.E. Romero-Herrera, and Genji Matsuda. 1979. Fitting the gene lineage into its species lineage. A parsimony strategy illustrated by cladograms constructed from globin sequences. Syst. Zool. 28, 2 (1979), 132-163.

[14] Paweł Górecki, Oliver Eulenstein, and Jerzy Tiuryn. 2013. Unrooted tree reconciliation: a unified approach. IEEE/ACM Trans. Comput. Biol. Bioinform. 10, 2 (2013), 522-36.

[15] Paweł Górecki and Jerzy Tiuryn. 2006. DLS-trees: A model of evolutionary scenarios. Theor. Comput. Sci. 359, 1-3 (2006), 378-399.

[16] Roderic Guigo, Ilya Muchnik, and Temple F Smith. 1996. Reconstruction of Ancient Molecular Phylogeny. Mol. Phylogenet. Evol. 6, 2 (1996), 189-213.

[17] Simon R. Harris et al. 2013. Whole-genome sequencing for analysis of an outbreak of meticillin-resistant Staphylococcus aureus: a descriptive study. Lancet Infect. Dis. 13, 2 (2013), 130-6.

[18] Ruth. A. Hufbauer, Robin A. Marrs, Aaron K. Jackson, René Sforza, Harsh Pal Bais, Jorge M. Vivanco, and Shannan E. Carney. 2003. Population structure, ploidy levels and allelopathy of Centaurea maculosa (spotted knapweed) and C. diffusa (diffuse knapweed) in North America and Eurasia. In Proceedings of the XI International Symposium on Biological Control of Weeds, Canberra Australia. USDA Forest Service. Forest Health Technology Enterprise Team, Morgantown, WV., 121-126.

[19] Andrew P. Jackson. 2004. A reconciliation analysis of host switching in plantfungal symbioses. Evolution 58, 9 (2004), 1909-23.

[20] Michael Lynch and John S. Conery. 2000. The evolutionary fate and consequences of duplicate genes. Science 290, 5494 (2000), 1151-5.

[21] Bin Ma, Ming Li, and Louxin Zhang. 2000. From Gene Trees to Species Trees. SIAM f. Comput. 30, 3 (2000), 729-752.

[22] Wayne P. Maddison. 1997. Gene Trees in Species Trees. Syst. Biol. 46 (1997), 523-536.

[23] Andrew P. Martin and Theresa M. M. Burg. 2002. Perils of paralogy: using HSP70 genes for inferring organismal phylogenies. Syst. Biol. 51, 4 (2002), 570-87.

[24] Fred R McMorris, David B Meronk, and Dean A Neumann. 1981. Consensusntrees. Bull. Math. Biol. 43, 2 (1981), 239-244.

[25] F. R. McMorris, D. B. Meronk, and D. A. Neumann. 1983. A View of Some Consensus Methods for Trees. Springer Berlin Heidelberg, Berlin, Heidelberg, 122-126.

[26] Jucheol Moon and Oliver Eulenstein. 2017. Synthesizing Species Trees from Unrooted Gene Trees: A Parameterized Approach. In Proceedings of the 8th ACM$B C B$. ACM, New York, NY, USA, 253-262.

[27] Serena Nik-Zainal et al. 2012. The life history of 21 breast cancers. Cell 149, 5 (2012), 994-1007.

[28] Susumu Ohno. 1970. Evolution by gene duplication. Springer-Verlag, Berlin.

[29] Roderic D.M. Page. 1998. GeneTree: comparing gene and species phylogenies using reconciled trees. Bioinformatics 14, 9 (1998), 819-820.

[30] Roderic D.M. Page. 2000. Extracting species trees from complex gene trees: reconciled trees and vertebrate phylogeny. Mol. Phylogenet. Evol. 14 (2000), 89106.

[31] Roderic D.M. Page and M. Charleston. 1997. Reconciled trees and incongruent gene and species trees. Math. Hierar. Biol. 37 (1997), 50 - 70.

[32] David F Robinson and Leslie R Foulds. 1979. Comparison of weighted labelled trees. In Combinatorial Mathematics VI, A.F. Horadam and W.D. Wallis (Eds.). Springer Berlin Heidelberg, Berlin, Heidelberg, 119-126.

[33] Fabian Schreiber et al. 2013. TreeFam v9: a new website, more species and orthology-on-the-fly. Nucleic Acids Res. 42, D1 (2013), D922-D925.

[34] André Wehe, J. Gordon Burleigh, and Oliver Eulenstein. 2013. Efficient algorithms for knowledge-enhanced supertree and supermatrix phylogenetic problems. IEEE/ACM Trans. Comput. Biol. Bioinform. 10, 6 (2013), 1432-41. 
Table 1: Majority cluster counts for the minimal trees inferred from the Guigó et al. dataset (trivial clusters are omitted). For example, under the RF cost the cluster counts over all optimal solution trees for this data set are 1291 for each of the clusters \{aves, mamm, rept $\}$ and \{aves, rept\}. Both of these clusters are strict consensus clusters since Figure 3 shows that there are 1291 optimal trees under the RF cost.

\begin{tabular}{|c|c|c|c|c|c|c|c|c|c|c|c|c|c|c|c|c|c|c|c|}
\hline D & $\mathrm{L} / \mathrm{DL}$ & DC & $\mathrm{RF}$ & 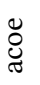 & $\underset{\widetilde{\sigma}}{\tilde{\sigma}}$ & $\begin{array}{l}\text { है } \\
\text { Е్ }\end{array}$ & $\begin{array}{l}\underset{\Xi}{\Xi} \\
\text { đ్త }\end{array}$ & 胥 & $\begin{array}{l}\stackrel{\infty}{d} \\
\stackrel{\varpi}{ \pm}\end{array}$ & 옹 & 胥 & تี & हैँ & $\stackrel{\infty}{\Xi}$ & 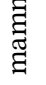 & $\begin{array}{l}\overline{\text { ह }} \\
\text { है }\end{array}$ & $\frac{⿱}{\tilde{\omega}}$ & $\begin{array}{l}\overrightarrow{0} \\
\overrightarrow{0}\end{array}$ & 苛 \\
\hline 71 & & & 1191 & + & + & + & + & + & + & + & + & + & + & + & + & + & + & & + \\
\hline 71 & 12 & 108 & 1091 & + & + & + & + & + & + & + & + & + & + & & + & + & + & & + \\
\hline 71 & 12 & 108 & 891 & + & + & + & + & + & + & & + & + & & & + & + & + & & + \\
\hline 71 & 12 & & & + & + & + & + & & + & & + & + & & & + & + & + & & + \\
\hline & & 108 & & & + & + & + & + & + & & + & + & & & + & + & + & & + \\
\hline & & 108 & & & + & + & + & & + & & + & & & & + & + & + & & + \\
\hline 45 & 10 & & & & + & + & & & + & & & + & & & + & + & + & & + \\
\hline & 7 & & & & + & + & & & + & & & & & & + & + & + & & + \\
\hline 71 & 12 & & & & + & + & & & + & & & & & & + & + & & & + \\
\hline & & $\begin{array}{l}100 \\
60\end{array}$ & 905 & & $\begin{array}{l}+ \\
+\end{array}$ & $\begin{array}{l}+ \\
+\end{array}$ & & & $\begin{array}{l}+ \\
+\end{array}$ & & & & & & $\begin{array}{l}+ \\
+\end{array}$ & & + & & $\begin{array}{l}+ \\
+\end{array}$ \\
\hline 71 & 12 & & & & + & & & & + & & & & & & + & + & & & + \\
\hline 71 & 12 & 68 & 703 & & + & & & & + & & & & & & + & & & & + \\
\hline & & 60 & & & & & + & & & & + & & & & & + & & & \\
\hline 71 & 12 & 108 & 1291 & & & & & & + & & & & & & + & & & & + \\
\hline 71 & 12 & 108 & 1291 & & & & & & + & & & & & & & & & & + \\
\hline 71 & 12 & 108 & 1191 & & & & & & & + & & & + & & & & & & \\
\hline & 8 & 68 & & + & & & $\begin{array}{l}+ \\
+\end{array}$ & & & & & & & & & + & & & \\
\hline
\end{tabular}

Table 2: Majority cluster counts counts for the maximal trees inferred from Guigó dataset. See also Figure 3 . The trivial clusters are omitted.

\begin{tabular}{|c|c|c|c|c|c|c|c|c|c|c|c|c|c|c|c|c|}
\hline Cost/Count & $\begin{array}{l}\ddot{0} \\
\text { J }\end{array}$ & శ్రే & $\frac{\text { క్ }}{\text { : }}$ & ‡్ & $\underset{\tilde{z}}{\tilde{Z}}$ & 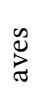 & 옹 & 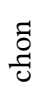 & $\overrightarrow{\mathcal{U}}$ & हैँ & $\stackrel{\infty}{\Xi}$ & $\begin{array}{l}\text { घี } \\
\text { త్ర్ }\end{array}$ & $\begin{array}{l}\bar{\Xi} \\
\text { ! }\end{array}$ & $\frac{u}{\tilde{0}}$ & $\begin{array}{l}\overrightarrow{0} \\
\overrightarrow{2}\end{array}$ & 苍 \\
\hline D 918676080 & + & + & + & + & + & & + & + & + & + & + & + & + & + & + & + \\
\hline $\begin{array}{l}\text { L } 42970800 \\
\text { L } 38896128\end{array}$ & $\begin{array}{l}+ \\
+\end{array}$ & $\begin{array}{l}+ \\
+\end{array}$ & $\begin{array}{l}+ \\
+\end{array}$ & $\begin{array}{l}+ \\
+\end{array}$ & $\begin{array}{l}+ \\
+\end{array}$ & & $\begin{array}{l}+ \\
+\end{array}$ & $\begin{array}{l}+ \\
+\end{array}$ & $\begin{array}{l}+ \\
+\end{array}$ & $\begin{array}{l}+ \\
+\end{array}$ & $\begin{array}{l}+ \\
+\end{array}$ & + & & $\begin{array}{l}+ \\
+\end{array}$ & $\begin{array}{l}+ \\
+\end{array}$ & $\begin{array}{l}+ \\
+\end{array}$ \\
\hline $\begin{array}{l}\text { DL } 1824912 \\
\text { DL } 1666224 \\
\text { DL } 1147296\end{array}$ & $\begin{array}{l}+ \\
+ \\
+\end{array}$ & $\begin{array}{l}+ \\
+ \\
+\end{array}$ & + & $\begin{array}{l}+ \\
+ \\
+\end{array}$ & $\begin{array}{l}+ \\
+ \\
+\end{array}$ & & $\begin{array}{l}+ \\
+ \\
+\end{array}$ & $\begin{array}{l}+ \\
+ \\
+\end{array}$ & $\begin{array}{l}+ \\
+\end{array}$ & $\begin{array}{l}+ \\
+ \\
+\end{array}$ & $\begin{array}{l}+ \\
+ \\
+\end{array}$ & $\begin{array}{l}+ \\
+ \\
+\end{array}$ & & $\begin{array}{l}+ \\
+ \\
+\end{array}$ & $\begin{array}{l}+ \\
+ \\
+\end{array}$ & \\
\hline $\begin{array}{l}\text { DC } 42278400 \\
\text { DC } 38896128\end{array}$ & $\begin{array}{l}+ \\
+\end{array}$ & $\begin{array}{l}+ \\
+\end{array}$ & $\begin{array}{l}+ \\
+\end{array}$ & $\begin{array}{l}+ \\
+\end{array}$ & $\begin{array}{l}+ \\
+\end{array}$ & & $\begin{array}{l}+ \\
+\end{array}$ & $\begin{array}{l}+ \\
+\end{array}$ & $\begin{array}{l}+ \\
+\end{array}$ & $\begin{array}{l}+ \\
+\end{array}$ & $\begin{array}{l}+ \\
+\end{array}$ & + & & $\begin{array}{l}+ \\
+\end{array}$ & $\begin{array}{l}+ \\
+\end{array}$ & $\begin{array}{l}+ \\
+\end{array}$ \\
\hline RF 11315838951936 & + & + & + & + & + & + & + & + & + & + & + & & + & + & + & + \\
\hline
\end{tabular}

[35] Louxin Zhang. 2011. From gene trees to species trees II: species tree inference by minimizing deep coalescence events. IEEE/ACM Trans. Comput. Biol. Bioinform. $8,6(2011), 1685-91$ 


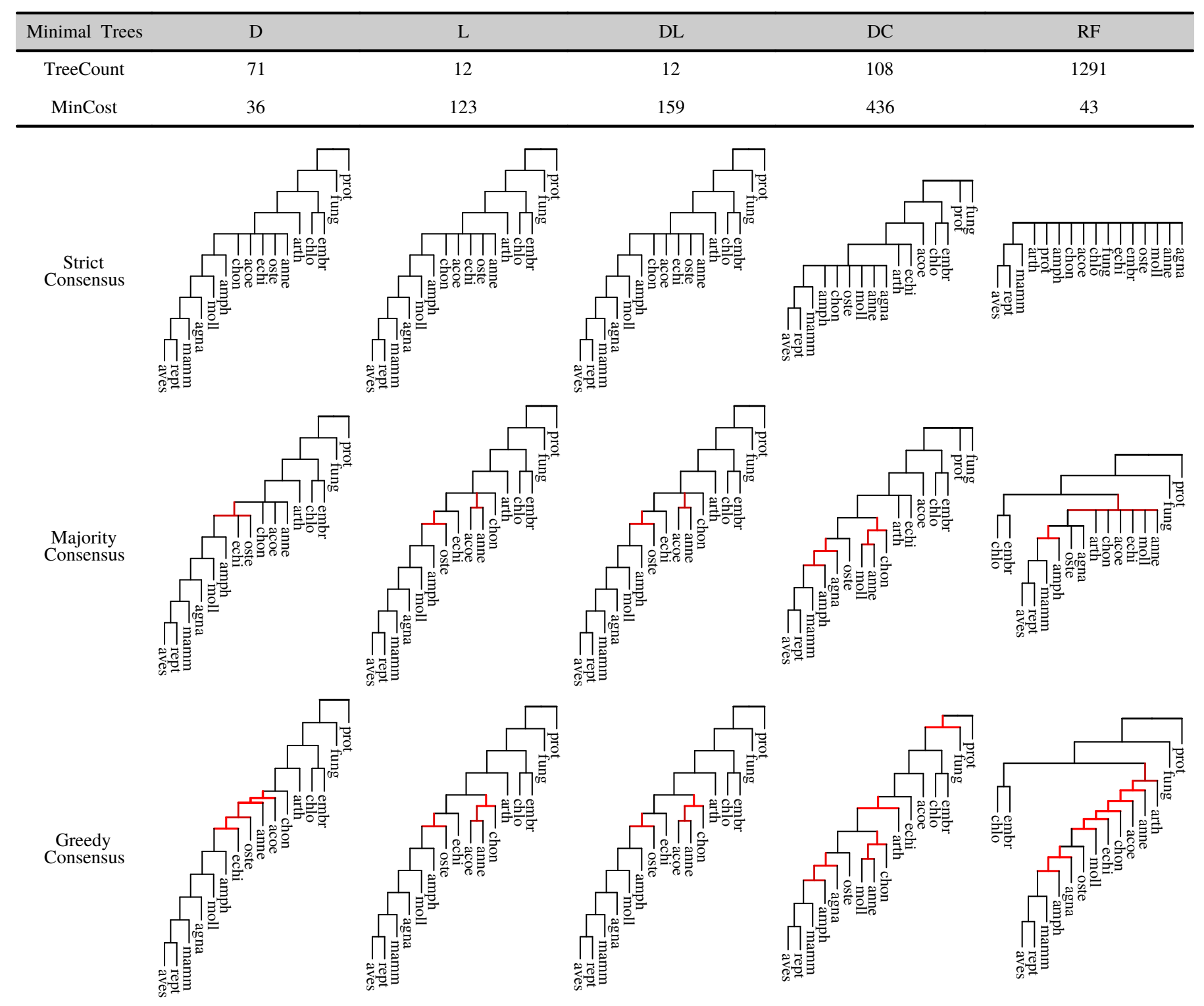

Figure 3: Consensus trees from optimal solutions to Guigó dataset [16]. Supports are indicated by the branch length and color. Well supported (> 70\%) edges are black. 


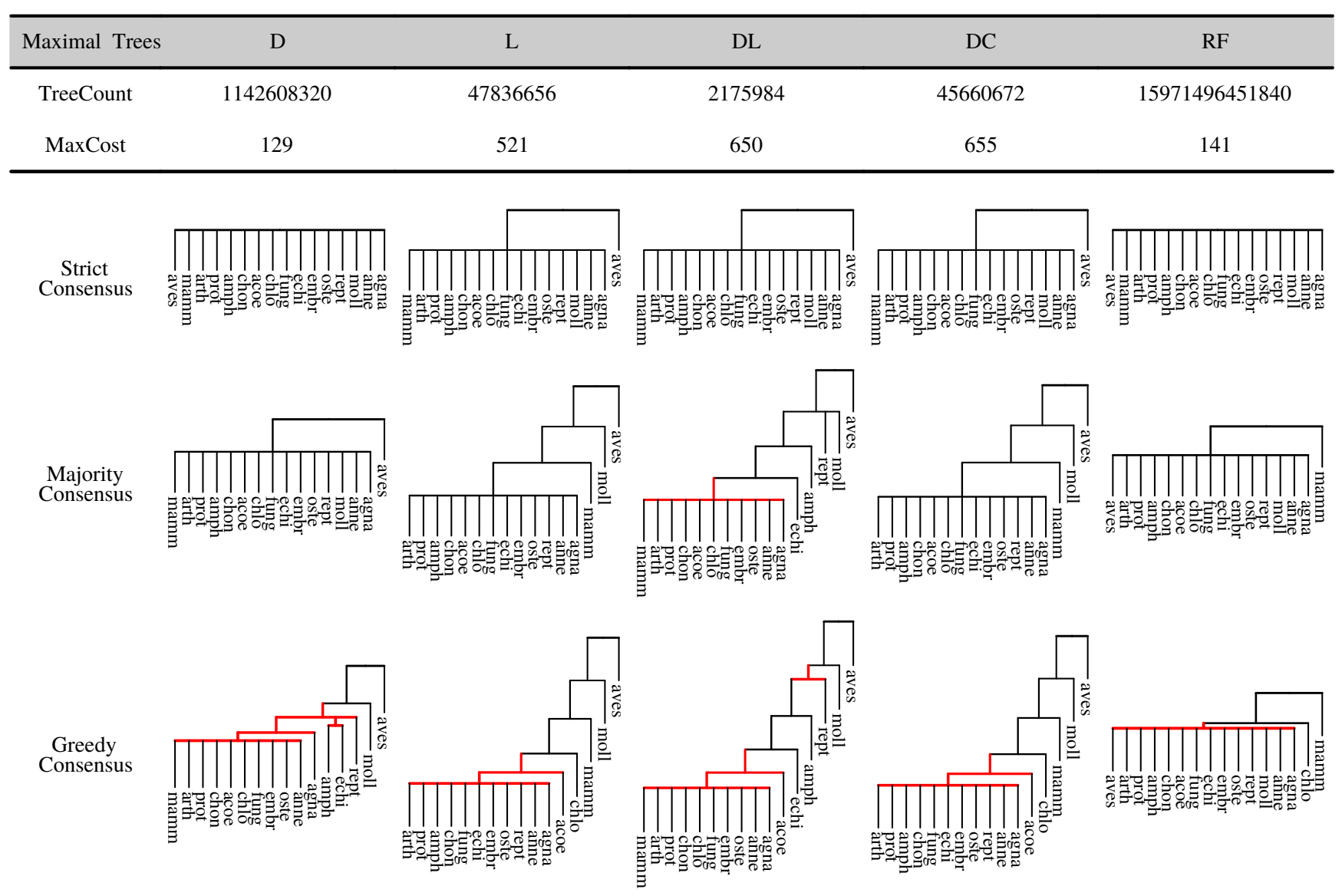

Figure 4: Consensus trees from the maximal trees inferred from Guigó dataset [16]. See also consensus trees from minimal trees in Figure 3. 